\title{
Important queries for the airway analysis in cone-beam computed tomography scans: Threshold tool and voxel size protocol
}

\author{
Luize Severo Martins, Gabriela Salatino Liedke', Heraldo Luis Da Silveira Dias², Priscila Fernanda Da Silveira Tiecher², \\ Nadia Assein Arus², Edwin M. Ongkosuwito³, Mariana Boessio Vizzotto² \\ Department of Stomatology, School of Dentistry, Federal University of Santa Maria, Santa Maria, Brazil, 'Department of Surgery and \\ Orthopedics, School of Dentistry, Federal University of Rio Grande do Sul, Porto Alegre, ${ }^{2}$ Department of Orthodontics and Craniofacial \\ Biology, Radboud Medical Center, ${ }^{3}$ Department of Orthodontics and Craniofacial Biology, Unit of the Radboud University Nijmegen Medical \\ Centre, Nijmegen, Netherlands
}

\section{A B S T R A C T}

Context: There is an expansion of the use of cone-beam computed tomography (CBCT) for maxillofacial diagnosis. However, some researchers have demonstrated inconsistencies between the results of airway analysis tools. Aim: This study aims to analyze the threshold tool presented in postprocessing software for airway volume estimation and the influence of voxel size in these measurements. Methods: Three hundred and sixteen-selected CBCT scans $(0.2,0.25$, and 0.4 voxel sizes) were retrospectively analyzed. A trained and calibrated examiner performed the volume measurements in specific sites in upper airway at 25 and chosen threshold tool using the Dolphin Software. Statistical Analysis Used: Analysis of variance (ANOVA) was used to compare the thresholds for each voxel and the differences between the preset and the chosen thresholds, while paired $t$-test to compare differences between the chosen thresholds for voxel size groups. Results: The threshold values range from 26 to 43 . The mean of the threshold selected for voxel 0.4 was significantly lower than the mean thresholds of $0.2 \mathrm{~mm}$ to $0.25 \mathrm{~mm}$ voxel. Small volumes were obtained with the preset threshold tool for all voxel sizes when compared with the chosen threshold. The mean of differences in volumes between preset and chosen threshold decreased with the increase of voxel size. Conclusion: The voxel size protocol influenced the threshold value choice for volume measurements in upper airway analysis. The thresholds near to 30 seem better filling the airway space.

Key words: Cone-beam computed tomography, dentistry, software tool, three-dimensional imaging, upper airway

\section{INTRODUCTION}

The expansion of cone-beam computed tomography (CBCT) yielded multiple benefits for dental and maxillofacial diagnosis. ${ }^{[1-6]}$ In addition to hard tissues analysis, CBCT scans allow the visualization of soft-tissue boundaries and airway spaces. Besides linear and area measures, some software offers a specific tool for the airway reconstruction

\begin{tabular}{|l|l|}
\hline \multicolumn{2}{|c|}{ Access this article online } \\
\hline Quick Response Code: & \\
\hline & Website: \\
\hline & www.joomr.org \\
& DOI: \\
\hline
\end{tabular}

and evaluation. This semiautomatic segmentation permits the users to determine the region of interest and adjust image threshold, according to the visual perception, which expands or reduces the software sensitivity to fill the airway space and result in estimated volume. Some researchers have shown controversial results about the consistency

\footnotetext{
This is an open access journal, and articles are distributed under the terms of the Creative Commons Attribution-NonCommercial-ShareAlike 4.0 License, which allows others to remix, tweak, and build upon the work non-commercially, as long as appropriate credit is given and the new creations are licensed under the identical terms.

For reprints contact: reprints@medknow.com

Cite this article as: Martins LS, Liedke GS, Da Silveira Dias HL, Da Silveira Tiecher PF, Arus NA, Ongkosuwito EM, et al. Important queries for the airway analysis in cone-beam computed tomography scans: Threshold tool and voxel size protocol. J Oral Maxillofac Radiol 2018;6:26-30.
}

Address for correspondence: Dr. Mariana Boessio Vizzotto, School of Dentistry Federal University of Rio Grande Do Sul, Oral Radiology Section, Ramiro Barcelos Street, 2492-5 ${ }^{\text {th }}$ Floor, Porto Alegre, Brazil. E-mail: mariana.vizzotto @ ufrgs.br 
Martins, et al.: Airway analysis in cone-beam computed tomography scans

when evaluating airway estimation tools, as well as the phantom utilized. ${ }^{[7-11]}$

For cephalometric and airway analysis, a large field of view (FOV) is frequently chosen to include all regions of interest. Usually, bigger voxel sizes are selected for large FOV's because it requires less scan and reconstruction time, and therefore less radiation for the patient; however, larger voxel sizes reduce spatial resolution. ${ }^{[9,12,13]}$ In this view, it is essential to understand the way an image threshold tool and scan protocol can influence volume measurements. Thus, the aim of this study was to analyze the threshold tool presented in postprocessing software for airway volume estimation and the influence of voxel size in this process.

\section{Methods}

This research protocol was approved by the Ethics Committee in the Federal University of Rio Grande do Sul (n 25300). The images used in this study were obtained from a database. The study sample consisted of 316 scans. The inclusion criteria were scans acquired with large FOV. All CBCT images were obtained with an i-CAT (Imaging Sciences International, Hatfield, PA) as part of the diagnostic records for clinical patients. The scans were acquired as follows: 250 scans with $0.2 \mathrm{~mm}$ voxel size, protocol (scanning protocol: $120 \mathrm{kV}, 5 \mathrm{~mA}$, $13 \mathrm{~cm} \times 17 \mathrm{~cm}$ FOV, scanning time of $40 \mathrm{~s}$ ); 30 scans with $0.25 \mathrm{~mm}$ voxel size (scanning protocol: $120 \mathrm{kV}, 5 \mathrm{~mA}$, $13 \mathrm{~cm} \times 17 \mathrm{~cm}$ FOV, scanning time of $40 \mathrm{~s}$ ); and 36 scans with $0.4 \mathrm{~mm}$ voxel size (scanning protocol: $120 \mathrm{kV}, 5 \mathrm{~mA}$, $13 \mathrm{~cm} \times 17 \mathrm{~cm}$ FOV, scanning time of $20 \mathrm{~s}$ ).

All images were evaluated using the "airway tool" available on Dolphin three-dimensional (3D) software (version 13.8, Dolphin Imaging and Management Solutions, Chatsworth, California). Intra- and inter-examiner calibration (L. S. M. and M. B. V.) were performed for volume measures (ICC >0.9) and threshold choice. The scans were analyzed by one calibrated examiner (L. S. M.). Before the measurements, the patient's head was aligned with the midsagittal plane perpendicular and the palatal plane parallel to the ground. The airway limits were defined: anterior border, a vertical plane from the posterior nasal spine (PNS) through up to skull basis, and the inferior border was a horizontal plane (parallel to ANS-PNS) at the superior point of the epiglottis. The volume from each CBCT image was calculated with two values of the threshold tool from Dolphin software: a preset sensitivity of 25 (available when the airway tool opens) and a sensitivity
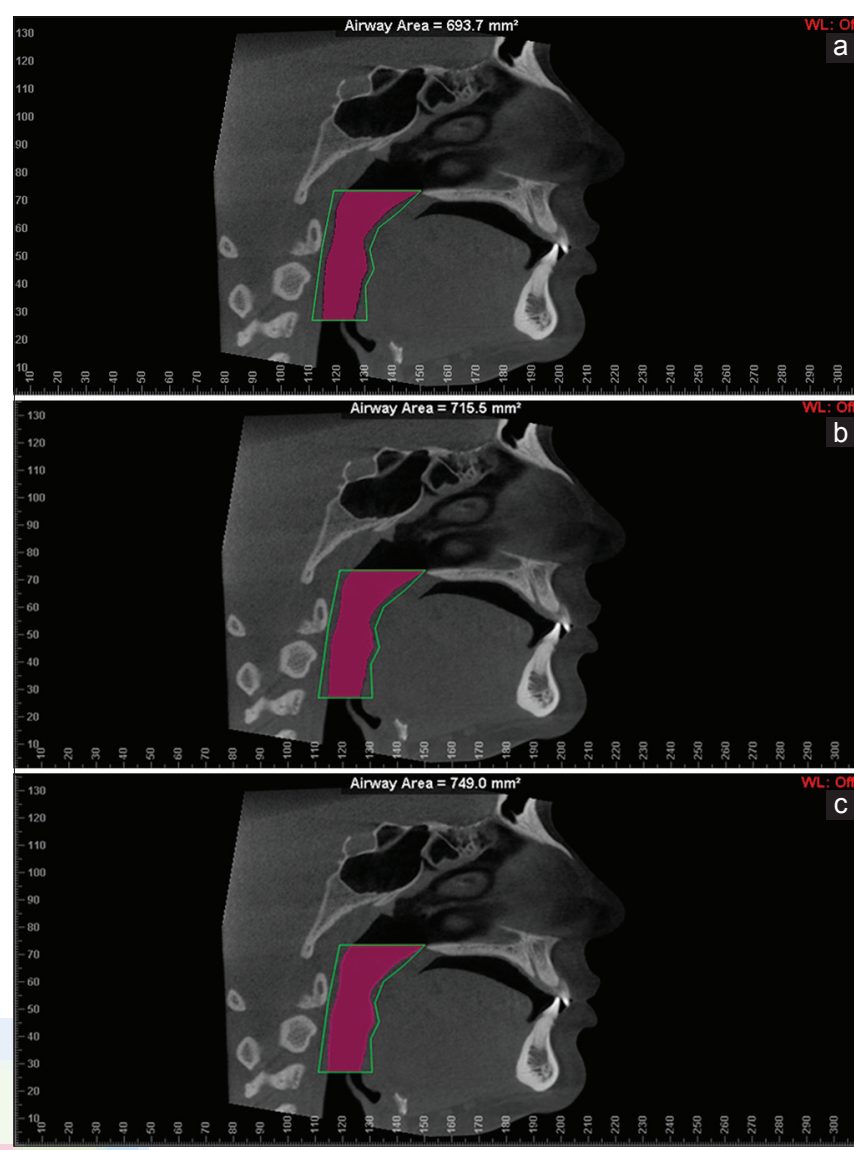

Figure I: Borders definition to measure the oropharynx volume using the threshold tool in Dolphin Software. (a) 25 preset threshold. (b) chosen threshold (35 in this scan) and (c) 70 threshold value

chosen by the examiner as the most compatible for the optimal filling of each airway space in the multiplanar analysis [Figure 1].

The statistical analysis was computed using SPSS software (version 17.0; SPSS, Chicago, Illinois, USA). The mean and range for airway volume were calculated for each voxel and threshold used. Analysis of variance (ANOVA) was used to compare the thresholds values for each voxel group. Paired samples $t$-test was used to compare differences between the chosen thresholds for voxel size groups. The level of statistical significance was $P<0.05$. ANOVA Welch analysis, complemented by Bonferroni post hoc test $(P<0.000)$, was used to compare the mean of differences among the voxel size groups.

\section{ResUlts}

Table 1 shows the frequency, percentages, quartiles, and median values for the chosen thresholds for each voxel size. The median of the chosen threshold increased as the voxel size of the image decreased. Table 2 shows that the mean of the threshold value selected for voxel 0.4 was significantly 
Martins, et al.: Airway analysis in cone-beam computed tomography scans

Table I: Frequency, percentages and median for thresholds selected in airway volume measures

\begin{tabular}{|c|c|c|c|c|c|c|c|c|}
\hline \multicolumn{3}{|c|}{$0.2 \mathrm{~mm}$ voxel size } & \multicolumn{3}{|c|}{$0.25 \mathrm{~mm}$ voxel size } & \multicolumn{3}{|c|}{$0.4 \mathrm{~mm}$ voxel size } \\
\hline Threshold & Frequency & Percent & Threshold & Frequency & Percent & Threshold & Frequency & Percent \\
\hline 26 & 0 & 0 & 26 & 0 & 0 & 26 & 6 & 16.7 \\
\hline 27 & 18 & 7.2 & 27 & 3 & 10 & $\underline{27} *$ & 14 & 38.9 \\
\hline 28 & 29 & 11.6 & 28 & 5 & 16.7 & $\overline{28}$ & 10 & 27.8 \\
\hline 29 & 48 & 19.2 & $\underline{29}$ & 8 & 26.7 & 29 & 4 & II.I \\
\hline 30 & 49 & 19.6 & 30 & 3 & 10 & 30 & 1 & 2.8 \\
\hline 31 & 40 & 16 & 31 & 10 & 33.3 & 31 & 1 & 2.8 \\
\hline 32 & 35 & 14 & 32 & 0 & 0 & 32 & 0 & 0 \\
\hline 33 & 24 & 9.6 & 33 & 0 & 0 & 33 & 0 & 0 \\
\hline 34 & 4 & 1.6 & 34 & I & 3.3 & 34 & 0 & 0 \\
\hline 35 & 2 & 0.8 & 35 & 0 & 0 & 35 & 0 & 0 \\
\hline 43 & I & 0.4 & 43 & 0 & 0 & 43 & 0 & 0 \\
\hline Total & 250 & 100.0 & Total & 30 & 100.00 & Total & 36 & 100.00 \\
\hline
\end{tabular}

Bold=Percentiles 25,50 (median) and 75. *Percentile 25 and 50

Table 2: Comparison of chosen thresholds among the voxels protocols analyzed in this study

\begin{tabular}{lccccc}
\hline \multicolumn{5}{c}{ Chosen thresholds } \\
Voxel & Mean & SD & SE & Minimum & Maximum \\
\hline 0.2 & 30,244 A & 1,9941 & 0,1261 & 27 & 43 \\
0.25 & 29,567 A & 1,6121 & 0,2943 & 27 & 34 \\
0.4 & 27,528 B & 1,1585 & 0,1931 & 26 & 31 \\
\hline
\end{tabular}

Different letters in same column indicate statistical difference tested under ANOVA and Bonferroni test $(P<0.05)$. SD: Standard deviation; SE: Standard error; ANOVA:Analysis of variance

lower than the mean thresholds of voxel $0.2 \mathrm{~mm}$ to $0.25 \mathrm{~mm}$. Table 3 shows mean and range for total airway volume calculated with preset and chosen thresholds in each voxel size. A paired samples $t$-test indicated statistically small volumes obtained with the preset threshold for all voxel sizes studied when compared with the chosen threshold. Table 4 shows the mean difference between the chosen and preset threshold, indicating that the values decrease with the increase of voxel size.

\section{Discussion}

The use of CBCT increases in dentistry, but specified protocols for airway analysis are not well established. [3,5,14-18] Some studies evaluated the airway space using various software and tools to calculate the volume. $\mathrm{El}$ and Palomo ${ }^{[7]}$ evaluated three commercially available software packages: Dolphin 3D (Dolphin Imaging and Management Solutions, Chatsworth, Calif), InVivoDental (Anatomage, San Jose, Calif), and OnDemand 3D (CyberMed, Seoul, Korea) and showed that the Dolphin 3D presented high reliability, but poor accuracy. The authors also noted that the software exhibited inconsistencies within themselves. Since the gray values on CBCT images do not correspond to the Hounsfield units from multislice computed tomography, it is not possible to perform image estimation according to each tissue. Therefore, to adjust soft-tissue boundaries all voxels are put together, and its gray values are used to render the surface disclosure. ${ }^{[19]}$
Yamashina et al., ${ }^{[10]}$ using VGStudio MAX1.2.1 software, evaluated the reliability and accuracy of CBCT using a phantom to measure the air, water, and soft-tissues density and concluded that the measurement of the airway volume was accurate. In our study, all images were analyzed using Dolphin software, which is widely used and provides a specific airway tool for area and volume estimations. To access the airway analysis, a semiautomatic segmentation is presented, in which the user should establish the soft-tissue borders and then locate the seed points into the airway space. A threshold tool is available so that the examiner can change the airway space-filling degree according to visual inspection. Since there is no standard protocol for these instruments and measurements, ${ }^{[8,14,20-22]}$ the calibrated observer calculated with the preset (25) and the best value that visually could fill the airway borders.

Alves $e$ al.$^{[8]}$ aimed to determine the most accurate threshold value for airway volume quantification based on an airway prototype. The authors evaluated different threshold values and suggested that the volumes measured with the threshold of 25 and 50 had statistically significant differences from the gold standard, and volumes measured with values from 70 to 75 showed no statistical differences from the gold standard and among them. The best thresholds values of this research ranged between 26 and 43, median of 30,29 , and 27 for $0.2 \mathrm{~mm}, 0.25 \mathrm{~mm}$, and $0.4 \mathrm{~mm}$ voxel sizes, respectively, diverging from the former study. In this research, a threshold of 70 or more clearly trespassed the soft-tissues boundaries, and consequently, the measurements were discarded. Our results showed statistical differences between the volume using the minimum value of threshold (25) and the observer chosen value, thus suggesting that maintenance of the preset threshold may underestimate the airway size. Furthermore, increasing the threshold resulted in an increased airway volume measured. This study has a limitation that there is not a gold standard 
Martins, et al.: Airway analysis in cone-beam computed tomography scans

Table 3: Upper airway volume and range $\left(\mathrm{mm}^{3}\right)$ in each voxel size group for preset (25) and chosen threshold

\begin{tabular}{lcccc} 
& $\mathbf{0 . 2}$ voxel size & $\mathbf{0 . 2 5}$ voxel size & $\mathbf{0 . 4}$ voxel size \\
\hline Mean and range for 25 threshold & $19602.8(7444.3-59056.30)$ A & $21698.90($ II $1768.40-34867.20)$ A & $22845.98($ I249I.70-42969.70) A \\
Mean and range for chosen threshold & $20637.65(7990.70-60105.10)$ B & $22626.31($ (I2097.80-36032.00) B & $23396.33($ (13042.70-43994.00) B \\
\hline
\end{tabular}

Different letters in same column indicate statistical difference tested under paired samples $t$-test $(P<0.05)$

Table 4: Upper airway volume $\left(\mathrm{mm}^{3}\right)$ and standard deviation in each voxel size group for preset (25) and chosen threshold

\begin{tabular}{lcccc}
\hline $\begin{array}{l}\text { Voxel } \\
\text { size }\end{array}$ & $\begin{array}{c}\text { Mean of } \\
\text { differences }\end{array}$ & $\begin{array}{c}\text { Minimum of } \\
\text { differences }\end{array}$ & $\begin{array}{c}\text { Maximum } \\
\text { differences }\end{array}$ & SD \\
\hline 0.2 & I034.84A & 109.4 & 4059 & 564.48 \\
0.25 & 927.4IA & 226.3 & 2734.1 & 516.72 \\
0.4 & 550.35B & 127.9 & 2119 & 359.10 \\
\hline
\end{tabular}

Different letters in same column indicate statistical difference tested under Welch complemented by Bonferroni test $(P<0.000)$. SD: Standard deviation

to determine the ideal threshold number for each voxel protocol, but on the other hand, the authors examined a huge number of patient's complementary examinations in contrast to a phantom.

The benefits and risks when requesting a CBCT scan should always be considered. ${ }^{[5,14]}$ Evidence-based guidelines for radiation protection outline rules for justification and optimization of CBCT exposures suggest individual protocols for different clinical situations. For orthodontics, the committee states "research is needed to define robust guidance on clinical selection for large-volume CBCT in orthodontics, based on quantification of benefit to patient outcome." ${ }^{[13]}$ In this sense, the voxel size determines the image resolution and should be selected according to the diagnostic task. Some protocols have a higher resolution (smaller voxel sizes) but also result in greater exposure to radiation for patient. ${ }^{[13,20]}$ It is prudent that the least needed resolution should be used to reduce patient exposure to radiation. This study compared the airway volume acquired with three voxel resolutions $-0.2 \mathrm{~mm}$, $0.25 \mathrm{~mm}$, and $0.4 \mathrm{~mm}$. When the mean differences of preset and chosen values of thresholds were assessed, the differences decreased with the increase in voxel size suggesting that the threshold choice varies on the voxel size, and both play a role in the airway volume measurement.

\section{Conclusion}

For airway assessment when using Dolphin Software, the thresholds values near to 30 showed better filling to the airway space. Using the preset threshold is not recommended since it might underestimate the airway values. Moreover, the acquisition protocol, specifically the voxel size, influenced the threshold choice and volume assessment. In vitro studies, trying to simulate the airway borders in phantoms should be executed to define the protocols, and consequently, the airway tools parameters to evaluate the volume in CBCT.

\section{Financial support and sponsorship Nil.}

\section{Conflicts of interest}

There are no conflicts of interest.

\section{References}

1. Kapila SD, Nervina JM. CBCT in orthodontics: Assessment of treatment outcomes and indications for its use. Dentomaxillofac Radiol 2015;44:20140282.

2. Hatcher DC. Cone beam computed tomography: Craniofacial and airway analysis. Dent Clin North Am 2012;56:343-57.

3. Horner K, O'Malley L, Taylor K, Glenny AM. Guidelines for clinical use of CBCT: A review. Dentomaxillofac Radiol 2015;44:20140225.

4. Holberg C, Steinhäuser S, Geis P, Rudzki-Janson I. Cone-beam computed tomography in orthodontics: Benefits and limitations.J Orofac Orthop 2005;66:434-44.

5. van Vlijmen OJ, Kuijpers MA, Bergé SJ, Schols JG, Maal TJ, Breuning H, et al. Evidence supporting the use of cone-beam computed tomography in orthodontics. J Am Dent Assoc 2012;143:241-52.

6. Kaur S, Rai S, Kaur M. Comparison of reliability of lateral cephalogram and computed tomography for assessment of airway space. Niger J Clin Pract 2014;17:629-36.

7. El H, Palomo JM. Measuring the airway in 3 dimensions: A reliability and accuracy study. Am J Orthod Dentofacial Orthop 2010;137:S50. e1-9.

8. Alves M Jr., Baratieri C, Mattos CT, Brunetto D, Fontes Rda C, Santos JR, et al. Is the airway volume being correctly analyzed? Am J Orthod Dentofacial Orthop 2012;141:657-61.

9. Da Silveira PF, Fontana MP, Oliveira HW, Vizzotto MB, Montagner F, Silveira HL, et al. CBCT-based volume of simulated root resorption - Influence of FOV and voxel size. Int Endod J 2015;48:959-65.

10. Yamashina A, Tanimoto K, Sutthiprapaporn P, Hayakawa Y. The reliability of computed tomography (CT) values and dimensional measurements of the oropharyngeal region using cone beam CT: Comparison with multidetector CT. Dentomaxillofac Radiol 2008;37:245-51.

11. de Oliveira MV, Wenzel A, Campos PS, Spin-Neto R. Quality assurance phantoms for cone beam computed tomography: A systematic literature review. Dentomaxillofac Radiol 2017;46:20160329.

12. Farman AG. ALARA still applies. Oral Surg Oral Med Oral Pathol Oral Radiol Endod 2005;100:395-7.

13. SEDENTEXCT. Radiation Protection: Cone Beam CT For Dental and Maxillofacial Radiology; 2012. Available from: http://www.sedentexct. eu/files/guidelines_final.pdf. [Last accessed on 2016 Jul 10].

14. Scarfe WC. "All that glitters is not gold": Standards for cone-beam computerized tomographic imaging. Oral Surg Oral Med Oral Pathol Oral Radiol Endod 2011;111:402-8.

15. Ye N, Li J, Lai W. Evaluating airway volume with CBCT. Am J Orthod 
Martins, et al.: Airway analysis in cone-beam computed tomography scans

Dentofacial Orthop 2012;142:284.

16. Tsolakis IA, Venkat D, Hans MG, Alonso A, Palomo JM. When static meets dynamic: Comparing cone-beam computed tomography and acoustic reflection for upper airway analysis. Am J Orthod Dentofacial Orthop 2016;150:643-50.

17. Zimmerman JN, Lee J, Pliska BT. Reliability of upper pharyngeal airway assessment using dental CBCT: A systematic review. Eur J Orthod 2017;39:489-96.

18. Alsufyani NA, Flores-Mir C, Major PW. Three-dimensional segmentation of the upper airway using cone beam CT: A systematic review. Dentomaxillofac Radiol 2012;41:276-84.

19. Pauwels R, Araki K, Siewerdsen JH, Thongvigitmanee SS. Technical aspects of dental CBCT: State of the art. Dentomaxillofac Radiol
2015;44:20140224.

20. Matta RE, von Wilmowsky C, Neuhuber W, Lell M, Neukam FW, Adler W, et al. The impact of different cone beam computed tomography and multi-slice computed tomography scan parameters on virtual three-dimensional model accuracy using a highly precise ex vivo evaluation method. J Craniomaxillofac Surg 2016;44:632-6.

21. Aboudara CA, Hatcher D, Nielsen IL, Miller A. A three-dimensional evaluation of the upper airway in adolescents. Orthod Craniofac Res 2003;6 Suppl 1:173-5.

22. Anandarajah S, Abdalla Y, Dudhia R, Sonnesen L. Proposal of new upper airway margins in children assessed by CBCT. Dentomaxillofac Radiol 2015;44:20140438. 